\title{
The low carriage prevalence of pneumococcus among community-dwelling older people: A cross-sectional study in Japan
}

\section{Ikkoh Yasuda $^{\mathrm{a}, \mathrm{b}}$, Motoi Suzuki ${ }^{\mathrm{a}, \mathrm{c}}$, Bhim Gopal Dhoubhadel ${ }^{\mathrm{d}}$, Mayumi Terada ${ }^{\mathrm{a}, \mathrm{e}}$, Akira Satoh ${ }^{\mathrm{f}}$, Eiichiro Sando ${ }^{a}$, Tomoko Hiraoka ${ }^{\mathrm{a}, \mathrm{b}}$, Masaki Kurihara ${ }^{\mathrm{g}}$, Nobuo Matsusaka ${ }^{\mathrm{g}}$, Fumitaka Kawahara ${ }^{\mathrm{h}}$, Koya Ariyoshi ${ }^{\mathrm{a}, \mathrm{b}, \mathrm{d}}$, Konosuke Morimoto ${ }^{\mathrm{a}, \mathrm{b}, \mathrm{e}, *}$}

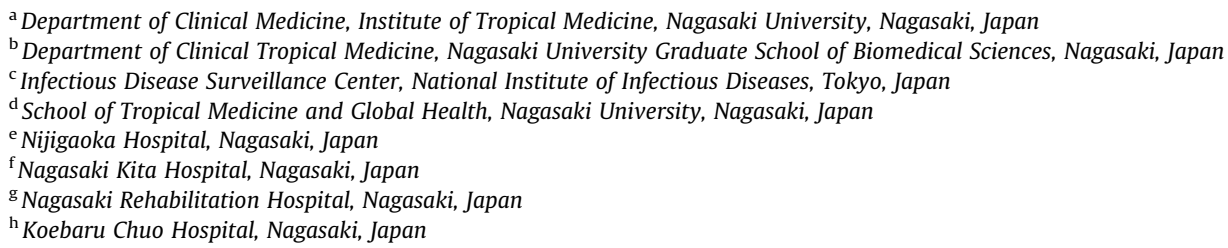

\section{A R T I C L E I N F O}

\section{Article history:}

Received 3 December 2019

Received in revised form 22 February 2020

Accepted 17 March 2020

Available online 4 April 2020

\section{Keywords:}

Streptococcus pneumoniae

Pneumococcus

Carriage prevalence

Community-dwelling

Older people

Superaged society

\begin{abstract}
A B S T R A C T
Background: The carriage prevalence of pneumococcus among community-dwelling older adults is not fully understood, especially in superaged societies. Our purpose was to elucidate the carriage prevalence of pneumococcus in the upper respiratory tract among Japanese community-dwelling adults aged $\geq 65$ years.

Methods: We conducted a cross-sectional study of generally healthy community-dwelling adults aged $\geq 65$ years in Nagasaki city, Japan. Demographic and clinical data and nasopharyngeal, oropharyngeal and saliva samples were collected from February 21st, 2018, to December 17th, 2018. The specimens were tested by culture and molecular methods.

Results: Among a total of 504 enrolled participants, none were positive for pneumococcus by culture, and 22 were positive by PCR. The overall carriage prevalence was 4.4\% (95\% CI: $2.8-6.5 \%$ ). The prevalence was highest in saliva samples, followed by oropharyngeal and nasopharyngeal samples. No demographic characteristics were associated with carriage prevalence, including age (4.7\% among participants aged $65-74$ years and $4.1 \%$ among those 75 years and older). Among the pneumococcal-positive participants, 18.2\% were PCV13-covered serotypes.

Conclusions: Our data suggest a low carriage prevalence of S. pneumoniae among community-dwelling older people in Japan.
\end{abstract}

(c) 2020 Elsevier Ltd. All rights reserved.

\section{Introduction}

Streptococcus pneumoniae (S. pneumoniae), or pneumococcus, is a causative agent of pneumococcal diseases, including pneumonia

Abbreviations: IPD, invasive pneumococcal disease; lytA, pneumococcal autolysin gene; lytA-qPCR, quantitative PCR for pneumococcal autolysin gene; NP, nasopharyngeal; OP, oropharyngeal; OR, odds ratio; PCR, polymerase chain reaction; PCV13, the 13-valent pneumococcal conjugate vaccine; PPV23, the 23-valent pneumococcal polysaccharide vaccine; qPCR, quantitative PCR; S. pneumoniae, Streptococcus pneumoniae; STGG, skim milk-tryptone-glucoseglycerol; URT, upper respiratory tract; $95 \% \mathrm{CI}, 95 \%$ confidence interval.

* Corresponding author at: Department of Clinical Medicine, Institute of Tropical Medicine, Nagasaki University, 1-12-4 Sakamoto, Nagasaki 852-8523, Japan.

E-mail address: komorimo@nagasaki-u.ac.jp (K. Morimoto). and invasive pneumococcal disease (IPD). Pneumococcal diseases continue to be a leading cause of morbidity and mortality globally, and the burdens of the diseases remain high, especially among older people [1-4]. Japan is a country with a growing superaged society, with the highest aging rate in the world; $28.1 \%$ of its population was aged 65 and over as of 2018 [5,6], and the burdens of pneumococcal diseases have been increasing among older people as the proportion of this population grows $[2,7]$.

Nasopharyngeal (NP) pneumococcal colonization at the mucosa of the upper respiratory tract (URT) is considered a source of transmission between hosts and an initial stage of the development pneumococcal disease [8]. Increased bacterial loads in the nasopharynx promote pneumococcal transmission to other individuals through bacterial shedding in nasal secretions [9] and 
increase the likelihood of progression to localized or invasive pneumococcal diseases (IPD), such as otitis media, pneumonia and meningitis, in the carrier [10].

The prevalence of pneumococcal carriage in children has been well studied. It is clearly higher than that in adults, as up to approximately half of children are pneumococcal carriers [1114]. Among community-dwelling older people, however, few reports are available on the pneumococcal carriage prevalence in the context of an aging society.

The objective of this study was to investigate the prevalence of pneumococcal carriage among community-dwelling older people in Japan.

\section{Material and methods}

\subsection{Study design and settings}

A cross-sectional study was performed from February 21st, 2018, to December 17th, 2018, in Nagasaki City, Japan. Community-dwelling older people aged $\geq 65$ years were enrolled. We enrolled them at regular clinic visit or outpatient rehabilitation visits at 4 hospitals in Nagasaki city: Nagasaki Rehabilitation Hospital, Koebaru Chuo Hospital, Nijigaoka Hospital and Nagasaki Kita Hospital. Their accompanying persons were also recruited if they were eligible.

In Japan, the PCV7 was introduced in 2010 for immunization of children $<5$ years old; the PCV7 was replaced by the PCV13 as a routine immunization for children in 2013. Among adults, the PCV13 was licensed for adults $\geq 65$ years old, and the 23 -valent pneumococcal polysaccharide vaccine (PPV23) was included in the routine immunization program for adults $\geq 65$ years old and 60-64 years old with specific medical conditions in 2014 [15]. The estimated vaccination coverage rate of the PCV13 in children was $97.4 \%$ in 2016 [16], and the coverage rate of the PPV23 among people 65 years old was estimated to be approximately $40.8 \%$ in 2015 [17].

\subsection{Eligibility criteria and data collection}

The inclusion criteria were people aged $\geq 65$ years who were ambulatory and not bedridden. The exclusion criteria were people with fever, pharyngodynia or any symptoms of URT infection; people who received antibiotic treatment in the previous 30 days; and people who stayed in the hospital or an elderly nursing home for $\geq 7$ days in the previous 30 days. The inclusion and exclusion criteria were applied to guarantee the generally healthy or well-controlled systemic disease status of participants. We collected the demographic and clinical information of participants, including age, sex, underlying disorders, facility visit frequency, family composition, care-need level and PPV23/PCV13 vaccination history. The care-need level was recorded in accordance with the Japanese long-term care insurance system classification as "Independent," "Support required" or "Care required" according to disablement (from the least to most disabled) [18,19].

\subsection{Sample collection}

NP, oropharyngeal (OP) and saliva samples were collected from each participant by researchers or trained research nurses. The details are as follows.

Two swab samples for culture were collected from each patient: one sample from the nasopharynx using a flocked swab (Opti-Swab LA-116, Puritan Medical Products Company, USA) and another sample from the oropharynx using a flocked swab (Opti-Swab LA-106, Puritan Medical Products Company, USA).
We used LA-116, which is thinner than LA-106, for nasopharyngeal sampling to promote participant comfort. The flocked swabs were immediately individually placed in $1 \mathrm{ml}$ of modified Amies media. Similarly, two swab samples for molecular analyses were collected from each patient: one sample from the nasopharynx using a flexible aluminum shaft swab with a rayon tip (Eiken Chemical Co., Tokyo, Japan) and another sample from the oropharynx using a wooden shaft swab with a rayon tip (Eiken Chemical Co., Tokyo, Japan). These flexible aluminum and wooden shaft swabs were immediately individually placed in $1 \mathrm{ml}$ skim milk-tryptoneglucose-glycerol (STGG) media. Saliva was collected with a sterile screw sputum processor (Eiken Chemical Co., Tokyo, Japan) and divided for culture and molecular analyses. NP swabbing techniques were performed in accordance with the World Health Organization (WHO) standard procedure [20]. OP swab samples were collected from the posterior pharyngeal wall. Collected sample media and saliva were transported to the laboratory within $5 \mathrm{~h}$ on ice and stored in a refrigerator for $3 \mathrm{~h}$ at most.

\subsection{Laboratory procedure}

Samples from the nasopharynx and oropharynx in Amies medium and saliva were examined by semiquantitative culture methods. Samples were plated on sheep blood agar (Nissui Pharmaceutical Co., Tokyo, Japan) with Isodisk CN (Alfresa Pharma Corporation, Osaka, Japan), which contained $35 \mu \mathrm{g} /$ disk of colistin and $60 \mu \mathrm{g} / \mathrm{disk}$ of nalidixic acid. Agar plates were incubated overnight at $35 \pm 0.5^{\circ} \mathrm{C}$ in $5 \% \mathrm{CO}_{2}$. Moreover, to verify the culture results, one additional oropharynx sample was consecutively collected from each last-quarter participant $(n=133)$ and tested with the culture method by another researcher separately.

NP and OP samples in STGG medium and residual saliva were further tested by quantitative PCR for the pneumococcal autolysin gene (lytA-qPCR). The details of the method have been described previously [21]. In brief, DNA was extracted from all the samples using a QIAamp DNA minikit (Qiagen GmbH, Hilden, Germany) according to the manufacturer's instructions. The pneumococcal autolysin gene (lytA) was screened in all the samples by lytA-qPCR, and samples were considered positive for S. pneumoniae when the detected lytA-specific signal with a $\leq 30$ cycle threshold $\left(C_{T}\right)$; specificity was confirmed by a melting curve analysis. All lytA-positive samples underwent an in-house nanofluidic realtime PCR assay to detect a total of 50 serotypes, including all the PCV13 and PPV23 serotypes [22,23]. Serogroup 18 was listed as a PCV13 vaccine type because this serogroup included all $18 \mathrm{~F} / 18 \mathrm{~A} / 18 \mathrm{~B} / 18 \mathrm{C} ; 18 \mathrm{C}$ is a vaccine serotype, and our assay did not differentiate them.

\subsection{Endpoint}

The primary endpoint was the pneumococcal carriage prevalence.

\subsection{Statistical analysis}

Considering an estimated 5\% pneumococcal carriage prevalence among older people according to previous studies, a sample of size $n=457$ would ensure that a $95 \%$ confidence interval $(95 \% \mathrm{CI})$ estimate of the prevalence was within $\pm 2 \%$, with $80 \%$ power.

All clinical, epidemiological and microbiological data were entered into an electronic database and analyzed by the investigator group. "Carriage prevalence" was defined as the total prevalence demonstrated from at least one NP and/or OP and/or saliva sample. One saliva sample from one participant could not be collected due to difficulty in collection, and the saliva result was considered negative in calculating the carriage prevalence. 
The carriage prevalence of pneumococcus was demonstrated with 95\% CIs by sampling site and detection method. The association between carriage and baseline characteristics was assessed by estimating odds ratios (ORs) with 95\% CIs using logistic regression models and were compared using Fisher's exact tests or $\chi^{2}$ tests when appropriate. We used Stata version 14.2 for all analyses (Stata Corp., College Station, TX, USA).

\subsection{Ethics}

This study was approved by the institutional review board of the Institute of Tropical Medicine, Nagasaki University, Nagasaki, Japan, and the institutional review boards of each study facility. The study was fully explained to all participants, and written informed consent was obtained from all participants or their families. We used anonymized data for the study analysis.

\section{Results}

\subsection{Baseline characteristics}

During the study period, a total of 504 participants aged $\geq 65$ years were enrolled. All sample collection was completed except one saliva sample from one participant.

The characteristics of the 504 enrolled subjects are summarized in Table 1. The mean and median age were 77.5 and 77.0 years old, respectively (range: 65.0 to 98.5 ), and 257 (51.0\%) were female. A total of 488 (96.8\%) had any underlying disorders, and the most common underlying disorder was cerebrovascular disease (294, $58.3 \%)$. A total of 89 (17.7\%) reported living alone, 267 (53.0\%) lived with one other person, and $4(0.8 \%)$ lived with children $\leq 5$ years old in the same household. Regarding the care-need level, 245 (48.6\%) were independent, 72 (14.3\%) required support, and 187 (37.1\%) required care. A total of 217 (43.1\%) participants reported having received the PPV23 within 5 years, and $2(0.4 \%)$ had received the PCV13. The accompanying persons accounted for less than $6.0 \%$ of all participants.

\subsection{Carriage prevalence of S. Pneumoniae}

The carriage prevalence of $S$. pneumoniae is summarized in Table 2. All NP, OP and saliva samples collected from the 504 participants were negative for $S$. pneumoniae by culture; therefore, the carriage prevalence of $S$. pneumoniae by culture method was $0.0 \%$ (95\% CI: $0.0-0.7 \%$ ). The lytA-qPCR assays revealed a total of 23 positive samples for $S$. pneumoniae in 22 participants: 1 NP sample (0.2\%, 95\% CI: $0.0-1.1 \%), 2$ OP samples $(0.4 \%, 95 \%$ CI: $0.0-1.4 \%)$ and 20 saliva samples $(4.0 \%, 95 \% \mathrm{CI}: 2.4-6.1 \%)$. Therefore, the carriage prevalence of $S$. pneumoniae by molecular analysis was $4.4 \%$ (95\% CI: $2.8-6.5 \%)$. There was monthly variation in carriage prevalence (2.1-10.0\%); however, clear seasonality was not observed.

\subsection{Serotypes of detected S. Pneumoniae}

Each serotype of the 23 lytA-positive samples from 22 participants was identified by nanofluidic real-time PCR, and the results are summarized in Table 3. Serotype $23 \mathrm{~F}$ was detected in both NP and saliva samples from the same participant. Four (18.2\%) of the 22 participants carried a pneumococcal serotype included in the PCV13, indicating a PCV13 vaccine-type carriage prevalence of $0.8 \%$ in the total participants. Multiple serotypes were identified in $11(50.0 \%)$ out of 22 lytA-positive participants.
Table 1

Characteristics of the participants.

\begin{tabular}{|c|c|}
\hline & All participants $(\mathrm{n}=504)$ \\
\hline \multicolumn{2}{|l|}{ Sex } \\
\hline Male & $247(49.0 \%)$ \\
\hline Female & 257 (51.0\%) \\
\hline \multicolumn{2}{|l|}{ Age group (years) } \\
\hline $65-74$ & $214(42.5 \%)$ \\
\hline$\geq 75$ & $290(57.5 \%)$ \\
\hline \multicolumn{2}{|l|}{ Residency } \\
\hline Home & 497 (98.6\%) \\
\hline Supportive housing & $7(1.4 \%)$ \\
\hline \multicolumn{2}{|l|}{ Underlying disorders } \\
\hline Any disorders & $488(96.8 \%)$ \\
\hline None & $16(3.2 \%)$ \\
\hline Chronic respiratory disease & $68(13.5 \%)$ \\
\hline No chronic respiratory disease & $436(86.5 \%)$ \\
\hline Cerebrovascular disease & $294(58.3 \%)$ \\
\hline No cerebrovascular disease & $210(41.7 \%)$ \\
\hline Other disorders & $280(55.6 \%)$ \\
\hline No other disorders & $224(44.4 \%)$ \\
\hline \multicolumn{2}{|c|}{ Facility visit frequency (per month) } \\
\hline$<1$ & $142(28.2 \%)$ \\
\hline $1-5$ & $190(37.7 \%)$ \\
\hline$>5$ & $172(34.1 \%)$ \\
\hline \multicolumn{2}{|l|}{ No. of people per household } \\
\hline 1 & $89(17.7 \%)$ \\
\hline 2 & $267(53.0 \%)$ \\
\hline$\geq 3$ & $148(29.4 \%)$ \\
\hline \multicolumn{2}{|l|}{ No. of children $\leq 5$ y.o. } \\
\hline 0 & $500(99.2 \%)$ \\
\hline 1 & $3(0.6 \%)$ \\
\hline 2 & $1(0.2 \%)$ \\
\hline \multicolumn{2}{|l|}{ Care-need level } \\
\hline Independent & $245(48.6 \%)$ \\
\hline Support required & $72(14.3 \%)$ \\
\hline Care required & $187(37.1 \%)$ \\
\hline \multicolumn{2}{|l|}{ PPV23 vaccination within 5 years } \\
\hline Not vaccinated & $258(51.2 \%)$ \\
\hline Vaccinated & $217(43.1 \%)$ \\
\hline No record & $29(5.8 \%)$ \\
\hline \multicolumn{2}{|l|}{ PCV13 vaccination } \\
\hline Not vaccinated & $488(96.8 \%)$ \\
\hline Vaccinated & $2(0.4 \%)$ \\
\hline No record & $14(2.8 \%)$ \\
\hline
\end{tabular}

Data are presented as n (\%). PPV23: 23-valent pneumococcal polysaccharide vaccine, PCV13: 13-valent pneumococcal conjugate vaccine.

\subsection{Risk factors associated with carriage}

Risk factors for carriage of $S$. pneumoniae are summarized in a supplementary table. None of the studied variables, such as sex, age group, residency, number of people per household and number of children $\leq 5$ years old, were significantly associated with pneumococcal carriage.

\section{Discussion}

In this study, we identified a low carriage prevalence of S. pneumoniae among community-dwelling older people in Japan. To our knowledge, this is the first study to investigate the prevalence of pneumococcal carriage, focusing on community-dwelling older people in Japan. The data observed in this study are clinically and epidemiologically important because Japan has the highest aging rate in the world, and the experiences of Japan can serve as a precedent for other aging and aged societies that are experiencing a trend toward rapid aging.

The carriage prevalence of S. pneumoniae by molecular methods was $4.4 \%$ in this study. Although a significant risk factor for pneumococcal carriage was not identified in this study because of the sample size and low carriage prevalence, some implications can 
Table 2

Carriage prevalence of $S$. pneumoniae by sampling site and detection method.

\begin{tabular}{|c|c|c|c|c|c|c|c|c|}
\hline & \multicolumn{2}{|c|}{$\begin{array}{l}\text { Nasopharyngeal } \\
(\mathrm{n}=504)\end{array}$} & \multicolumn{2}{|c|}{$\begin{array}{l}\text { Oropharyngeal } \\
(\mathrm{n}=504)\end{array}$} & \multicolumn{2}{|c|}{ Saliva $(n=503)$} & \multicolumn{2}{|c|}{$\begin{array}{l}\text { Carriage prevalence } \\
(\mathrm{n}=504)\end{array}$} \\
\hline & $\mathrm{n}$ & $\%(95 \% \mathrm{CI})$ & $\mathrm{n}$ & $\%(95 \% \mathrm{CI})$ & $\mathrm{n}$ & $\%(95 \% \mathrm{CI})$ & $\mathrm{n}$ & $\%(95 \% \mathrm{CI})$ \\
\hline Streptococcus pneumoniae (lytA-qPCR) & 1 & $0.2(0.0-1.1)$ & 2 & $0.4(0.0-1.4)$ & 20 & $4.0(2.4-6.1)$ & 22 & $4.4(2.8-6.5)$ \\
\hline Streptococcus pneumoniae (culture) & 0 & $0.0(0.0-0.7)$ & 0 & $0.0(0.0-0.7)$ & 0 & $0.0(0.0-0.7)$ & 0 & $0.0(0.0-0.7)$ \\
\hline
\end{tabular}

Data are presented as n, \% $(95 \% \mathrm{CI})$. "Carriage prevalence" is the total prevalence demonstrated from at least one NP and/or OP and/or saliva sample. One saliva sample from one participant could not be collected due to difficulty in collection, and the saliva result was considered negative in calculating the carriage prevalence. lytA-qPCR: quantitative PCR for the pneumococcal autolysin gene.

be observed. The child contact was low in this population because only $0.8 \%$ of our participants reported children $\leq 5$ years old in the same household, which may suggest reduced exposure to S. pneumoniae. According to previous research, the exposure to colonized children, who form the main reservoir of pneumococcus, is commonly assumed to be risk factor of carriage in adults [24-26]. Additionally, a reduction in vaccine-type carriage prevalence among nontarget populations was demonstrated after PCV introduction in a wide range of countries $[27,28]$. The PCV for children was introduced in 2010 in Japan, and the PCV13 vaccine type accounted for only a small proportion of the pneumococcal carriage prevalence in this study: $0.8 \%$ out of $4.4 \%$. Pediatric PCV immunization programs may have indirectly affected the carriage prevalence among the unvaccinated population. In other developed countries, a wide range of pneumococcal carriage prevalence rates have been reported in recent years. Two large-scale studies from Portugal and the U.S., respectively, suggested low carriage prevalence, consistent with our results among a similar population. In a study from Portugal, the carriage prevalence among adults aged $\geq 60$ years was $2.3 \%$ in $2010-2012$ [29]. A study from the U. S. reported a $1.8 \%$ carriage prevalence among noninstitutionalized adults aged $\geq 65$ years in 2015-2016 [30]. On the other hand, a large-scale study from the Netherlands reported a much higher carriage prevalence than those in the aforementioned studies, with the prevalence reaching 17.8-17.9\% among community-dwelling older adults aged $\geq 65$ years in $2008-2009$, shortly after the intro-

Table 3

Pneumococcal serotypes/group detected by molecular method (nanofluidic real-time PCR) in 22 pneumococcal carriers identified by lytA-qPCR.

\begin{tabular}{|c|c|c|c|c|c|}
\hline & Serotypes/group & Nasopharyngeal & Oropharyngeal & Saliva & Total \\
\hline \multicolumn{6}{|l|}{ (a) } \\
\hline \multirow[t]{7}{*}{ Single serotype } & $10 \mathrm{~A}$ & 0 & 0 & 2 & $2(9.1 \%)$ \\
\hline & $12 \mathrm{~F} / 12 \mathrm{~A} / 12 \mathrm{~B} / 44 / 46$ & 0 & 0 & 2 & $2(9.1 \%)$ \\
\hline & $35 \mathrm{~F}$ & 0 & 0 & 2 & $2(9.1 \%)$ \\
\hline & $23 \mathrm{~F}$ & $1^{*}$ & 0 & $1^{*}$ & $1(4.5 \%)$ \\
\hline & $33 \mathrm{~F} / 33 \mathrm{~A} / 37$ & 0 & 0 & 1 & $1(4.5 \%)$ \\
\hline & $35 \mathrm{~B}$ & 0 & 0 & 1 & $1(4.5 \%)$ \\
\hline & Nontypable & 0 & 1 & 1 & $2(9.1 \%)$ \\
\hline \multirow[t]{10}{*}{ Multiple serotypes } & $9 \mathrm{~N} / 9 \mathrm{~L}+10 \mathrm{~A}$ & 0 & 1 & 1 & $2(9.1 \%)$ \\
\hline & $2+17 F$ & 0 & 0 & 1 & $1(4.5 \%)$ \\
\hline & $5+10 A+35 B$ & 0 & 0 & 1 & $1(4.5 \%)$ \\
\hline & $6 \mathrm{~A} / 6 \mathrm{~B}+17 \mathrm{~F}$ & 0 & 0 & 1 & $1(4.5 \%)$ \\
\hline & $6 C / 6 D+35 F$ & 0 & 0 & 1 & $1(4.5 \%)$ \\
\hline & $9 \mathrm{~N} / 9 \mathrm{~L}+10 \mathrm{~A}+23 \mathrm{~A}$ & 0 & 0 & 1 & $1(4.5 \%)$ \\
\hline & $9 \mathrm{~N} / 9 \mathrm{~L}+15 \mathrm{~B} / 15 \mathrm{C}$ & 0 & 0 & 1 & $1(4.5 \%)$ \\
\hline & $10 \mathrm{~A}+12 \mathrm{~F} / 12 \mathrm{~A} / 12 \mathrm{~B} / 44 / 46$ & 0 & 0 & 1 & $1(4.5 \%)$ \\
\hline & $10 \mathrm{~A}+18^{* *}$ & 0 & 0 & 1 & $1(4.5 \%)$ \\
\hline & $10 \mathrm{~A}+34$ & 0 & 0 & 1 & $1(4.5 \%)$ \\
\hline \multicolumn{6}{|l|}{ (b) } \\
\hline \multirow[t]{4}{*}{ PCV13 vaccine type } & 5 & 0 & 0 & 1 & $1(4.5 \%)$ \\
\hline & $6 \mathrm{~A} / 6 \mathrm{~B}$ & 0 & 0 & 1 & $1(4.5 \%)$ \\
\hline & $18^{* *}$ & 0 & 0 & 1 & $1(4.5 \%)$ \\
\hline & $23 \mathrm{~F}$ & $1^{*}$ & 0 & $1^{*}$ & $1(4.5 \%)$ \\
\hline \multirow[t]{13}{*}{ Non-PCV13 vaccine type } & $10 \mathrm{~A}$ & 0 & 1 & 8 & $9(40.9 \%)$ \\
\hline & $9 \mathrm{~N} / 9 \mathrm{~L}$ & 0 & 1 & 3 & $4(18.2 \%)$ \\
\hline & $12 \mathrm{~F} / 12 \mathrm{~A} / 12 \mathrm{~B} / 44 / 46$ & 0 & 0 & 3 & $3(13.6 \%)$ \\
\hline & $35 \mathrm{~F}$ & 0 & 0 & 3 & $3(13.6 \%)$ \\
\hline & $17 \mathrm{~F}$ & 0 & 0 & 2 & $2(9.1 \%)$ \\
\hline & $35 B$ & 0 & 0 & 2 & $2(9.1 \%)$ \\
\hline & 2 & 0 & 0 & 1 & $1(4.5 \%)$ \\
\hline & $6 C / 6 \mathrm{D}$ & 0 & 0 & 1 & $1(4.5 \%)$ \\
\hline & $15 B / 15 C$ & 0 & 0 & 1 & $1(4.5 \%)$ \\
\hline & $23 \mathrm{~A}$ & 0 & 0 & 1 & $1(4.5 \%)$ \\
\hline & $33 F / 33 A / 37$ & 0 & 0 & 1 & $1(4.5 \%)$ \\
\hline & 34 & 0 & 0 & 1 & $1(4.5 \%)$ \\
\hline & Nontypable & 0 & 1 & 1 & $2(9.1 \%)$ \\
\hline
\end{tabular}

Data are presented as $\mathrm{n} . \mathrm{n}$ is the number of carriers positive for each serotype/group by sampling site. The distribution of single/multiple serotypes is shown in (a), and each serotype/group is individually shown in (b). "Total" is the total number of carriers positive for each serotype/group demonstrated from at least one NP and/or OP and/or saliva sample, and \% is the rate among the 22 carriers. Nontypable: The samples were not typable by our assay. ${ }^{*} 23 \mathrm{~F}$ was detected in both NP and saliva samples from the same participant. Because some participants had multiple serotypes, the total number of carriers by serotype/group in Table (b) does not add up to $22 .{ }^{* *}$ Serogroup 18 is listed as PCV13 vaccine type because this serogroup includes all $18 \mathrm{~F} / 18 \mathrm{~A} / 18 \mathrm{~B} / 18 \mathrm{C} ; 18 \mathrm{C}$ is a vaccine serotype, and our assay did not differentiate them. 
duction of the PCV7 for use in all newborns in 2006 [31,32]. These three studies used similar methodology, culture and molecular methods for NP and OP samples; hence, they are basically mutually comparable. Although the applied methods differed from those used in these three studies, the following two studies also reported informative carriage prevalence. A study from Germany conducted culture-based screening for nasal samples taken from both anterior nares and OP samples and detected no pneumococci among community dwellers aged $\geq 65$ years in 2012-2013 [33]. A study from Italy reported a relatively high rate of $9.8 \%$ among generally healthy adults aged $\geq 65$ years using solely a molecular method for OP samples in 2015 [34]. This wide range of percentages may indicate that pneumococcal carriage prevalence has social and geographical diversity; however, fair comparison is difficult because of inconsistent methodologies among studies, including the sampling site (NP and/or OP and/or saliva), detection method (culture-based detection and/or molecular detection), and target genes of molecular detection.

This study elucidated the pneumococcal carriage prevalence according to sampling site and detection method. S. pneumoniae was detected most frequently in saliva $(4.0 \%)$, followed by OP $(0.4 \%)$ and NP $(0.2 \%)$ samples using the molecular method, but it was not detected in any sampling sites using the culture method. Although the current gold standard recommended by the WHO basically in line with the recommended methodology for children, namely, the isolation of live $S$. pneumoniae by culture for NP specimens and additional OP specimens from adults if possible [20], the most appropriate methodology for carriage studies among adults is still controversial. Unlike children, anatomical changes in the adult URT can cause difficulty in accessing the NP niche, and low pneumococcal density or bacterial cocolonization makes pneumococcal identification by culture laborious and likely to result in low sensitivity [35]. Adoption of alternative sampling sites, such as the oropharynx and saliva, or detection methods, such as molecular methods to compensate for the low sensitivity of the current gold standard may prompt other concerns for further investigation. Saliva sampling was reported to significantly increase pneumococcal detection $[35,36]$. The microbially rich characteristics of saliva and OP samples, however, make pneumococcal detection on culture plates difficult or sometimes nearly impossible [37]. Additionally, applying molecular methods to saliva or OP samples may cause overestimation because of nonpneumococcal streptococci, such as S. mitis, S. oralis, and S. pseudopneumoniae in the oropharynx, which are not only morphologically highly similar to $S$. pneumoniae but even carry S. pneumoniae homolog genes $[30,38]$. Compared to nasopharyngeal carriage, the clinical significance of saliva carriage has yet to be determined, especially in terms of transmissibility and potentiality to cause disease [39], though there are a few reports about the clinical significance of saliva carriage: a high saliva carriage rate, the same as that of nasopharyngeal carriage, has been reported among schoolchildren based on a PCR-based method [40], and pneumococcal transmission via saliva has also been reported among young adults [41]. It is urgent to establish a standard methodology for the assessment of pneumococcal carriage prevalence in adults and older people. The clinical significance of each carriage location and the carriage density, such as transmissibility and potentiality to cause disease, should be considered.

Although older people are at high risk of pneumococcal diseases $[2,42]$, pneumococcal carriage was shown to be less common among older people in this and other studies, suggesting increasing disease susceptibility with reduced carriage prevalence. Possible hypotheses for this obvious discrepancy have been proposed: Age-associated inflammation contributes to bacterial invasion into host cells [43], infrequent colonization in older people is insufficient for boosting colonization-induced antibodies [39,44], and a mouse model suggested that ageing impairs innate mucosal immune response in the URT [45]. These age-related changes in immune function may cause pneumococcal acquisition to directly lead to disease progression with a relatively short carriage duration [46].

This study identified multiple serotypes in 11 (50.0\%) out of 22 lytA-positive participants. Cocolonization of more than one pneumococcal strain is considered to promote the acquisition of antibiotic resistance and capsule switch via horizontal gene transfer [47]. Although there are several reports regarding multiple serotype carriage among children [48], few reports are available on the situation of older people, and further investigations are needed $[35,30]$.

The strength of our study design is that we identified the carriage prevalence according to the sampling site (nasopharynx, oropharynx or saliva) and detection method (culture-based detection or molecular detection), which permits mutual comparisons with previous studies. However, there are several limitations of our study. This cross-sectional study lacked longitudinal data and could not document the incidence of pneumococcal acquisition. The sample size was calculated for estimation of pneumococcal prevalence, and as a result, it was insufficient for the risk factor analysis combined with low pneumococcal carriage prevalence. The participants who were enrolled at regular clinic visits or outpatient rehabilitation visits could induce selection bias because this population tends to have a greater need for care and more comorbidities, especially cerebrovascular disease, than healthy individuals. Recruiting accompanying persons may also introduce overestimation when they are living with other participants because their samples are not mutually independent. Nevertheless, the carriage prevalence determined by molecular methods was as low as $4.4 \%$, and we assume that the true carriage prevalence might be lower. Although we set 30 days as the antibiotics washout period, given the low carriage prevalence and limited exposure to pneumococcus, a longer antibiotics washout period might be appropriate in older people.

\section{Conclusions}

Our data demonstrate a low carriage prevalence of S. pneumoniae in the URT in community-dwelling older people in Japan. Further investigations are needed to elucidate the clinical and epidemiological significance of the low carriage prevalence.

\section{Ethics approval and consent to participate}

Ethical approval for this study was obtained from the institutional review board of the Institute of Tropical Medicine, Nagasaki University, Nagasaki, Japan (number: 171207185), and the institutional review boards of each study facility.

\section{Informed consent}

The study was fully explained to all participants, and written informed consent was obtained from all subjects or their representatives.

\section{Data statement}

The datasets analyzed in this study are available from the corresponding author upon reasonable request.

\section{Declaration of Competing Interest}

The authors declare the following financial interests/personal relationships which may be considered as potential competing interests: [Konosuke Morimoto reports receiving speaker fees from Pfizer and MSD. All other authors declare no competing interests.]. 


\section{Acknowledgments}

We thank Kazuko Arai for her contribution to sample collection. We thank Rina Shiramizu and Kyoko Uchibori for the technical assistance and Yumi Araki for the administrative work. We also wish to thank Chuken (Nagasaki, Japan) for microbiological laboratory testing. We are grateful to all the collaborators and staff at the participating hospitals.

\section{Funding}

This research was supported by Pfizer, Japan.

\section{Role of the funding source}

The funding sources had no role in the study design; the collection, analysis and interpretation of data; the writing of the report, or the decision to submit the article.

\section{Authors' contributions}

MS and KM proposed the study idea. IY, AS, MT, ES, TH, MS, and KM supervised the research nurse and staff to ensure the integrity of the study procedure and sample collection. BGD supervised the lytA-qPCR and nanofluidic real-time PCR assays. IY, MS and KM performed the analysis and interpreted the findings. IY drafted the first report. All authors contributed to the writing of the final report. All authors have approved the final version to be submitted.

\section{Appendix A. Supplementary material}

Supplementary data to this article can be found online at https://doi.org/10.1016/j.vaccine.2020.03.033.

\section{References}

[1] Wahl B, O’Brien KL, Greenbaum A, Majumder A, Liu L, Chu Y, et al. Burden of Streptococcus pneumoniae and Haemophilus influenzae type b disease in children in the era of conjugate vaccines: global, regional, and national estimates for 2000-15. Lancet Glob Health 2018;6:e744-57.

[2] Ubukata K, Takata M, Morozumi M, Chiba N, Wajima T, Hanada S, et al. Effects of pneumococcal conjugate vaccine on genotypic penicillin resistance and serotype changes, Japan, 2010-2017. Emerg Infect Dis 2018;24:2010-20.

[3] Jain S, Self WH, Wunderink RG, Fakhran S, Balk R, Bramley AM, et al. Community-acquired pneumonia requiring hospitalization among U.S. adults. N Engl J Med 2015;373:415-27.

[4] Welte T, Torres A, Nathwani D. Clinical and economic burden of communityacquired pneumonia among adults in Europe. Thorax 2012;67:71-9.

[5] Muramatsu N, Akiyama H. Japan: super-aging society preparing for the future. Gerontologist 2011;51:425-32.

[6] Cabinet Office, Government of Japan. Annual report on the ageing society; 2019 [accessed 21 February 2020]. https://www8.cao.go.jp/kourei/english/ annualreport/2019/pdf/2019.pdf.

[7] Morimoto K, Suzuki M, Ishifuji T, Yaegashi M, Asoh N, Hamashige N, et al. The burden and etiology of community-onset pneumonia in the aging Japanese population: a multicenter prospective study. PLoS ONE 2015;10:e0122247.

[8] Simell B, Auranen K, Kayhty H, Goldblatt D, Dagan R, O’Brien KL, et al. The fundamental link between pneumococcal carriage and disease. Expert Rev Vaccines 2012;11:841-55.

[9] Dhoubhadel BG, Yasunami M, Nguyen HA, Suzuki M, Vu TH, Thi Thuy Nguyen A, et al. Bacterial load of pneumococcal serotypes correlates with their prevalence and multiple serotypes is associated with acute respiratory infections among children less than 5 years of age. PLOS ONE 2014;9:e110777.

[10] Weiser JN, Ferreira DM, Paton JC. Streptococcus pneumoniae: transmission, colonization and invasion. Nat Rev Microbiol 2018;16:355-67.

[11] Bogaert D, De Groot R, Hermans PW. Streptococcus pneumoniae colonisation: the key to pneumococcal disease. Lancet Infect Dis 2004;4:144-54.

[12] Regev-Yochay G, Raz M, Dagan R, Porat N, Shainberg B, Pinco E, et al. Nasopharyngeal carriage of Streptococcus pneumoniae by adults and children in community and family settings. Clin Infect Dis 2004;38:632-9.

[13] Otsuka T, Chang B, Shirai T, Iwaya A, Wada A, Yamanaka N, et al. Individual risk factors associated with nasopharyngeal colonization with Streptococcus pneumoniae and Haemophilus influenzae: a Japanese birth cohort study. Pediatr Infect Dis J 2013;32:709-14.

[14] Yahiaoui RY, Den Heijer C, Van Bijnen EM, Paget WJ, Pringle M, Goossens H, et al. Prevalence and antibiotic resistance of commensal Streptococcus pneumoniae in nine European countries. Future Microbiol 2016;11:737-44.

[15] Sando E, Suzuki M, Furumoto A, Asoh N, Yaegashi M, Aoshima M, et al. Impact of the pediatric 13-valent pneumococcal conjugate vaccine on serotype distribution and clinical characteristics of pneumococcal pneumonia in adults: the Japan Pneumococcal Vaccine Effectiveness Study (J-PAVE). Vaccine 2019;37:2687-93.

[16] Sakiyama H, Joh A, Umemoto S, Shimizu H, Oishi K. Childhood vaccination rates in Japan based on national survey. J Ambulatory Gener Pediatr 2017;20:272-82.

[17] Murakami Y, Nishiwaki Y, Kanazu SI, Oba M, Watanabe A. A nationwide survey of PPSV23 vaccine coverage rates and their related factors among the elderly in Japan, 2016. Nihon Koshu Eisei Zasshi 2018;65:20-4.

[18] Tsutsui T, Muramatsu N. Care-needs certification in the long-term care insurance system of Japan. J Am Geriatr Soc 2005;53:522-7.

[19] Sugiyama T, Tamiya N, Watanabe T, Wakui T, Shibayama T, Moriyama Y, et al. Association of care recipients' care-need level with family caregiver participation in health check-ups in Japan. Geriatr Gerontol Int 2018;18:26-32.

[20] Satzke C, Turner P, Virolainen-Julkunen A, Adrian PV, Antonio M, Hare KM, et al. Standard method for detecting upper respiratory carriage of Streptococcus pneumoniae: updated recommendations from the World Health Organization Pneumococcal Carriage Working Group. Vaccine 2013:32:165-79.

[21] Dhoubhadel BG, Yasunami M, Nguyen HA, Suzuki M, Vu TH, Nguyen ATT, et al. Bacterial load of pneumococcal serotypes correlates with their prevalence and multiple serotypes is associated with acute respiratory infections among children less than 5 years of age. PLoS ONE 2014;9:e110777.

[22] Dhoubhadel BG, Yasunami M, Yoshida LM, Thi HA, Thi TH, Thi TA, et al. A novel high-throughput method for molecular serotyping and serotype-specific quantification of Streptococcus pneumoniae using a nanofluidic real-time PCR system. J Med Microbiol 2014;63:528-39.

[23] Suzuki M, Dhoubhadel BG, Ishifuji T, Yasunami M, Yaegashi M, Asoh N, et al. Serotype-specific effectiveness of 23-valent pneumococcal polysaccharide vaccine against pneumococcal pneumonia in adults aged 65 years or older: a multicentre, prospective, test-negative design study. Lancet Infect Dis 2017;17:313-21.

[24] Hausdorff WP, Feikin DR, Klugman KP. Epidemiological differences among pneumococcal serotypes. Lancet Infect Dis 2005;5:83-93.

[25] Hussain M, Melegaro A, Pebody RG, George R, Edmunds WJ, Talukdar R, et al. A longitudinal household study of Streptococcus pneumoniae nasopharyngeal carriage in a UK setting. Epidemiol Infect 2005;133:891-8.

[26] Hamaluba M, Kandasamy R, Ndimah S, Morton R, Caccamo M, Robinson H, et al. A cross-sectional observational study of pneumococcal carriage in children, their parents, and older adults following the introduction of the 7valent pneumococcal conjugate vaccine. Medicine (Baltimore) 2015;94:e335.

[27] Davis SM, Deloria-Knoll M, Kassa HT O'Brien KL. Impact of pneumococcal conjugate vaccines on nasopharyngeal carriage and invasive disease among unvaccinated people: review of evidence on indirect effects. Vaccine 2013;32:133-45.

[28] Loo JD, Conklin L, Fleming-Dutra KE, Knoll MD, Park DE, Kirk J, et al. Systematic review of the indirect effect of pneumococcal conjugate vaccine dosing schedules on pneumococcal disease and colonization. Pediatr Infect Dis J 2014;33(Suppl 2):S161-71.

[29] Almeida ST, Nunes S, Paulo ACS, Valadares I, Martins S, Breia F, et al. Low prevalence of pneumococcal carriage and high serotype and genotype diversity among adults over 60 years of age living in Portugal. PLoS ONE 2014;9:e90974.

[30] Milucky J, Carvalho MG, Rouphael N, Bennett NM, Talbot HK, Harrison LH, et al. Streptococcus pneumoniae colonization after introduction of 13-valent pneumococcal conjugate vaccine for US adults 65 years of age and older, 2015-2016. Vaccine 2019:37:1094-100.

[31] Van Deursen AMM, Van Houten MA, Webber C, Patton M, Scott D, Patterson S, et al. The impact of the 13-valent pneumococcal conjugate vaccine on pneumococcal carriage in the community acquired pneumonia immunization trial in adults (CAPITA) study. Clin Infect Dis 2018;67:42-9.

[32] Bonten MJ, Huijts SM, Bolkenbaas M, Webber C, Patterson S, Gault S, et al. Polysaccharide conjugate vaccine against pneumococcal pneumonia in adults. N Engl J Med 2015;372:1114-25.

[33] Drayss M, Claus H, Hubert K, Thiel K, Berger A, Sing A, et al. Asymptomatic carriage of Neisseria meningitidis, Haemophilus influenzae, Streptococcus pneumoniae, Group A Streptococcus and Staphylococcus aureus among adults aged 65 years and older. PLoS ONE 2019;14:e0212052.

[34] Esposito S, Mari D, Bergamaschini L, Orenti A, Terranova L, Ruggiero L, et al. Pneumococcal colonization in older adults. Immun Ageing 2016;13:2.

[35] Krone CL, Wyllie AL, Van Beek J, Rots NY, Oja AE, Chu ML, et al. Carriage of Streptococcus pneumoniae in aged adults with influenza-like-illness. PLoS ONE 2015;10:e0119875.

[36] Krone CL, Van de Groep K, Trzcinski K, Sanders EA, Bogaert D. Immunosenescence and pneumococcal disease: an imbalance in hostpathogen interactions. Lancet Respir Med 2014;2:141-53.

[37] Wyllie AL, Rumke LW, Arp K, Bosch AA, Bruin JP, Rots NY, et al. Molecular surveillance on Streptococcus pneumoniae carriage in non-elderly adults; little 
evidence for pneumococcal circulation independent from the reservoir in children. Sci Rep 2016;6:34888.

[38] Dewhirst FE, Chen T, Izard J, Paster BJ, Tanner AC, Yu WH, et al. The human ora microbiome. J Bacteriol. 2010;192:5002-17.

[39] Adler H, Ferreira DM, Gordon SB, Rylance J. Pneumococcal Capsular Polysaccharide Immunity in the Elderly. Clin Vaccine Immunol 2017:24.

[40] Wyllie AL, Chu ML, Schellens MH, van Engelsdorp Gastelaars J, Jansen MD, van der Ende A, et al. Streptococcus pneumoniae in saliva of Dutch primary school children. PLoS ONE 2014;9:e102045.

[41] Levine H, Zarka S, Dagan R, Sela T, Rozhavski V, Cohen DI, et al. Transmission o Streptococcus pneumoniae in adults may occur through saliva. Epidemio Infect 2012:140:561-5.

[42] Melegaro A, Edmunds WJ, Pebody R, Miller E, George R. The current burden of pneumococcal disease in England and Wales. J Infect 2006;52:37-48.

[43] Boyd AR, Orihuela CI. Dysregulated inflammation as a risk factor for pneumonia in the elderly. Aging Dis 2011:2:487-500.
[44] Ferreira DM, Neill DR, Bangert M, Gritzfeld JF, Green N, Wright AK, et al. Controlled human infection and rechallenge with Streptococcus pneumoniae reveals the protective efficacy of carriage in healthy adults. Am J Respir Crit Care Med 2013;187:855-64.

[45] Krone CL, Trzcinski K, Zborowski T, Sanders EA, Bogaert D. Impaired innate mucosal immunity in aged mice permits prolonged Streptococcus pneumoniae colonization. Infect Immun 2013;81:4615-25.

[46] Adler H, Ferreira DM, Gordon SB, Rylance J. Pneumococcal capsular polysaccharide immunity in the elderly. Clin Vaccine Immunol 2017;24:e00004-e17.

[47] Brugger SD, Frey P, Aebi S, Hinds J, Muhlemann K. Multiple colonization with S. pneumoniae before and after introduction of the seven-valent conjugated pneumococcal polysaccharide vaccine. PLoS ONE 2010;5:e11638.

[48] Kandasamy R, Gurung M, Thapa A, Ndimah S, Adhikari N, Murdoch DR, et al. Multi-serotype pneumococcal nasopharyngeal carriage prevalence in vaccine naive Nepalese children, assessed using molecular serotyping. PLoS ONE 2015; 10:e0114286. 